\title{
AVALIAÇÃO DAS COMPETÊNCIAS E OBJETIVOS DE APRENDIZAGEM DO CURSO DE ENGENHARIA CIVIL DO CCT/UDESC
}

DOI: 10.37702/2175-957X.COBENGE.2021.3487

Jennifer Perreira dos Santos - sjenniferp@gmail.com

Universidade do Estado de Santa Catarina

Rua Ibrahim Manoel Matos 90

89230-550 - Joinville - SC

Leonardo Romero Monteiro - leonardo.monteiro@udesc.br

Universidade do Estado de Santa Catarina

Rua Dom Bosco 155

89222-540 - Joinville - SC

SANDRA DENISE KRUGER ALVES - sandra.kruger@udesc.br

Universidade do Estado de Santa Catarina

RUA JOAO PESSOA 984

89218-280 - JOINVILLE - SC

Resumo: Atualmente, existe dificuldade em recrutar engenheiros que além do domínio técnico possuam habilidades relacionadas à liderança, trabalho em grupo, planejamento, gestão estratégica e aprendizado de forma autônoma. O presente trabalho busca descrever a situação atual do curso de Engenharia Civil do Centro de Ciências Tecnológicas da Universidade do Estado de Santa Catarina através do estabelecimento de objetivos de aprendizagem e sua relação com o desenvolvimento das competências desejadas ao perfil do egresso. Os resultados mostram que grande parte das competências desenvolvidas dentro das atividades estão voltadas para o conhecimento mais básico que juntas correspondem a $68 \%$ da recorrência das competências correspondentes nos objetivos de aprendizado, mesmo que apenas $30 \%$ da carga horária do curso seja destinada a disciplinas de formação básica. Existe pouca ocorrência do desenvolvimento de habilidades transversais, que equivale a $14 \%$ da carga horária total avaliada. Entende-se que as observações realizadas pela presente pesquisa podem ser confirmadas em outros cursos de diferentes instituições de ensino. 


\section{COBENCE $2021 \begin{aligned} & \text { eIV Simpósio Internacional } \\ & \text { de Educação em Engenharia } \\ & \text { da ABENGE }\end{aligned}$ 28 a 30 de SETEMBRO \\ Formação em Engenharia: Tecnologia, Inovaçáo e Sustentabilidade"}

Palavras-chave: Ensino em Engenharia. Diretrizes Curriculares Nacionais. Competências. 


\section{AVALIAÇÃO DAS COMPETÊNCIAS E OBJETIVOS DE APRENDIZAGEM DO CURSO DE ENGENHARIA CIVIL DO CCT/UDESC}

\section{INTRODUÇÃO}

A engenharia trabalha constantemente com a incerteza, com dados incompletos e demandas simultâneas de clientes, autoridades governamentais, grupos ambientais e o público em geral. Para isso, a profissão de engenheiro requer habilidades em relações humanas, tal qual necessita de competência técnica. Complementarmente, os engenheiros devem lidar com as relações comerciais no mundo moderno considerando as consequências legais de suas decisões (SCOTT; HADGRAFT; ILIC, 2003).

Diante disso, existe a necessidade de atualizar a formação da engenharia no país, visando atender as demandas futuras por mais e melhores engenheiros considerando a expectativa de parte da comunidade acadêmica, das empresas que dependem dessa mão de obra qualificada e dos setores que representam a atuação profissional da área. Entretanto, existem dificuldades no mercado em recrutar engenheiros que além do domínio técnico possuam as chamadas habilidades transversais (ou também soft skills) habilidades relacionadas à liderança, trabalho em grupo, planejamento, gestão estratégica e aprendizado de forma autônoma (CNE/CES, 2019).

Em virtude dessas necessidades, o ensino superior precisa diminuir sua as atividades em que o aluno é apenas um observador que depois será avaliado para uma abordagem mais participativa do aluno na aprendizagem, e uma questão principal a ser abordada é a aprendizagem centrada no aluno (KOLMOS et al., 2008). A concepção de competências constitui a base essencial no mundo profissional e, portanto, também é um elemento-chave de qualquer modelo educacional (DE LOS RÍOS-CARMENADO; LÓPEZ; GARCÍA, 2015).

Nesse sentido, é verificada uma grande distância entre o modelo educacional predominante no Brasil e aquele capaz de formar os profissionais que a sociedade necessita, como afirma Scallon (2017). A Resolução CNE (2019) esclarece que um currículo focado nas competências depreende a substituição da lógica da assimilação prévia dos conteúdos e posterior incorporação e uso, pela ocorrência simultânea dessa compreensão com o desenvolvimento de um conjunto de habilidades e atitudes originados dos conhecimentos específicos.

Com base nas informações apresentadas, o curso de Engenharia Civil do Centro de Ciências Tecnológicas (CCT) da Universidade do Estado de Santa Catarina (UDESC) iniciou os estudos para a atualização do Projeto Pedagógico do Curso (PPC) no início de 2020. Ao longo deste ano diversos dados foram levantados para que fosse possível compreender qual a situação atual do curso e como poderíamos aperfeiçoá-lo para que o PPC esteja de acordo com as Diretrizes Curriculares Nacionais (DCNs) de 2019 (CNE, 2019).

O presente trabalho busca descrever a situação atual do curso de engenharia civil da UDESC/CCT através do estabelecimento de objetivos de aprendizagem e sua relação com o desenvolvimento das competências desejadas, com base no atual PPC, implementado em 2008. A partir disto é possível definir com melhor embasamento as reformulações necessárias na estrutura curricular do curso para que as competências desejadas no egresso sejam efetivamente desenvolvidas. 


\section{O CURSO DE GRADUAÇÃO EM ENGENHARIA CIVIL DO CENTRO DE} CIÊNCIAS TECNOLÓGICAS DA UNIVERSIDADE DO ESTADO DE SANTA CATARINA

O atual curso de Engenharia Civil do campus de Joinville da UDESC (Centro de Ciências Tecnológicas) foi criado em 1979 e o projeto pedagógico vigente foi publicado em 2008 (DEC UDESC/CCT, 2008). O curso possui uma estrutura de 10 semestres, em que cada semestre é composto por 18 semanas e segue o sistema de créditos, onde cada crédito corresponde à uma hora-aula (h/a) de cinquenta minutos de duração. Segundo a proposta do PPC de 2018 o curso tem 4968 horas-aula (DEC UDESC/CCT, 2008).

A proposta do PPC de 2008 visou adequar o curso às Leis de Diretrizes e Bases, bem como reformular o curso para uma proposta mais atualizada, com a criação de duas ênfases e melhoria da grade curricular. Além disso, apresentou alterações na carga horária do curso passando a atender a Resolução CNE/CES n² 2, de 18 de junho de 2007 (DEC UDESC/CCT, 2008).

De acordo com este PPC, o curso objetiva desenvolver a consciência social e política do aluno, de forma a capacitá-lo para o exercício da cidadania plena. Além disso, também são objetivos do curso:

I. Proporcionar uma sólida formação em disciplinas básicas e profissionalizantes que habilitem o egresso a acompanhar o ritmo de desenvolvimento técnico-científico do setor e adaptar-se às transformações;

II. Estimular o espírito de iniciativa, inventividade, empreendedorismo e de liderança, capacitando o profissional como agente ativo das transformações sociais;

III. Estimular um bom relacionamento humano, com posturas éticas e de colaboração permanente;

IV. Desenvolver a capacidade de comunicação gráfica, oral e escrita, sabendo utilizar as ferramentas necessárias para um melhor desempenho das funções;

V. Colaborar com uma postura relacionada com o respeito ao meio ambiente, à segurança do trabalhador, e a sensibilidade relativa a aspectos sociais e a sustentabilidade.

Com o propósito de estar em consonância com as Diretrizes Curriculares Nacionais, buscou-se estabelecer metas específicas a serem observadas na organização do currículo. Assim, algumas metas propostas para a qualidade de formação apresentadas pelo PPC, pertinentes a esta pesquisa (DEC UDESC/CCT, 2008) são:

I. Reduzir o tempo em sala de aula sem, contudo, reduzir o conteúdo na mesma proporção;

II. Dar ao aluno mais responsabilidade e participação no processo de aprendizagem;

III. Propiciar ao curso um enfoque maior em laboratórios e projetos;

IV. Melhorar as habilidades nas relações interpessoais, de comunicação e expressão;

V. Possibilitar uma formação complementar de maior abrangência, com a possibilidade de escolha de duas ênfases (área municipal e área ambiental).

Além disso, seguindo os indicativos das DCNs de 2002 o curso deveria oferecer aos alunos:

I. Capacidade de resolver problemas de Engenharia Civil, o que implica no domínio de conceitos fundamentais para o pleno exercício da profissão;

II. Capacidade de elaborar, de forma independente e soberana, seus juízos de valor e de decidir sobre as questões técnicas de sua responsabilidade;

III. Desenvolvimento de uma base intelectual e técnica que possibilite ao profissional continuar aprendendo novos conceitos, técnicas e formalismos. 
O Quadro 1 mostra que a grade curricular para o Curso de Engenharia Civil atende as Diretrizes Curriculares Nacionais de 2002 em relação às porcentagens dos núcleos de conteúdos (DEC UDESC/CCT, 2008). Em questão às atividades complementares esclarece-se que possibilitam 0 reconhecimento das habilidades, conhecimentos e competências citadas pelas Diretrizes Nacionais Curriculares. A realização e a validação dessas atividades para a integralização de horas as resoluções dos conselhos superiores da UDESC. Assim, o estudante deverá cumprir um total de 396 horas-aula reconhecidas nos termos da Resolução 05/2006 do CONSEPE (DEC UDESC/CCT, 2008).

Quadro 1 -Quadro de atendimento às Diretrizes Curriculares Nacionais.

\begin{tabular}{|c|c|c|c|c|}
\hline & \multicolumn{3}{|c|}{ Currículo Proposto } & \multirow{2}{*}{ DCN, 2002 (C.H) } \\
\hline & Hora-aula & Créditos & $\%$ & \\
\hline Disciplinas do núcleo básico & 1476 & 82 & 34,17 & $30 \%$ \\
\hline $\begin{array}{l}\text { Disciplinas do núcleo } \\
\text { profissionalizante }\end{array}$ & 702 & 39 & 16,25 & $15 \%$ \\
\hline $\begin{array}{l}\text { Disciplinas do núcleo } \\
\text { específico }\end{array}$ & 2142 & 119 & 49,58 & $55 \%$ \\
\hline TOTAL PARCIAL & 4320 & 240 & 100 & \\
\hline $\begin{array}{c}\text { Trabalho de Conclusão de } \\
\text { Curso }\end{array}$ & 36 & 2 & 0,83 & Não estabelece. \\
\hline $\begin{array}{l}\text { Estágio Curricular } \\
\text { Supervisionado }\end{array}$ & 216 & 12 & 5,00 & 160 horas (mínimo) \\
\hline Atividades complementares & 396 & 22 & 9,17 & Não estabelece. \\
\hline TOTAL & 4968 & 276 & & \\
\hline
\end{tabular}

Fonte: Adaptado de PPC (2008).

Segundo o PPC (DEC UDESC/CCT, 2008), os instrumentos do processo de ensinoaprendizagem são aulas expositivas dialogadas, dinâmicas de grupos, estudos dirigidos, estudos de caso, seminários e grupos de verbalização, onde serão contempladas a compreensão, interpretação e análise dos exercícios propostos, bem como aulas práticas em laboratórios. Entretanto, não existe uma definição exata de como cada metodologia deve ser aplicada ou para quais disciplinas são sugeridas.

Ainda, a verificação da aprendizagem das disciplinas ministradas deverá contemplar um procedimento coerente de ações a serem desenvolvidas pelo professor que garantam a qualidade de ensino e condições efetivas para o planejamento do trabalho a ser desenvolvido pelo estudante (DEC UDESC/CCT, 2008). No PPC não se apresenta como que as disciplinas se relacionam com os objetivos do curso, não sendo possível, por instrumentos fornecidos pelo próprio PPC, uma avaliação de como os objetivos estão sendo alcançados.

\section{ANÁLISE DOS OBJETIVOS DE APRENDIZADO E COMPETÊNCIAS}

Entre novembro e dezembro de 2020 foi proposto ao Núcleo Docente Estruturante (NDE) a atividade de elencar as competências gerais do curso e habilidades transversais das disciplinas constituintes do atual PPC. Essa dinâmica envolveu a análise da DCN de 2019, do PPC do DEC, dos PPCs de outras IES e de documentos de órgãos de acreditação 
de curso, como a Accreditation Board for Engineering and Technology (ABET), resultando nas competências apresentadas no Quadro 2.

Quadro 2 - Competências do DEC reavaliadas.

\begin{tabular}{|c|l|c|}
\hline ID1 & Competências gerais do curso & Palavra-chave \\
\hline A1 & Aprender e aplicar conhecimentos de matemática, ciência e engenharia. & Conhecer \\
\hline A2 & $\begin{array}{l}\text { Analisar e compreender fenômenos físicos e químicos inerentes a Engenharia } \\
\text { Civil. }\end{array}$ & Compreender \\
\hline A3 & Projetar e conduzir experimentos, bem como analisar e interpretar dados. & Investigar \\
\hline A4 & Formular, analisar e resolver problemas de Engenharia Civil. & Resolver \\
\hline A5 & $\begin{array}{l}\text { Conceber soluções de Engenharia Civil, analisando e compreendendo a } \\
\text { necessidade dos usuários e seu contexto. }\end{array}$ & Soluções \\
\hline A6 & Implantar, supervisionar e controlar as soluções de Engenharia Civil. & Executar \\
\hline A7 & $\begin{array}{l}\text { Conhecer e aplicar com ética a legislação e os atos normativos compreendendo } \\
\text { o impacto das soluções nos setores global, econômico, ambiental e contexto } \\
\text { social. }\end{array}$ & Legislação \\
\hline A8 & $\begin{array}{l}\text { Criar, projetar e implementar soluções tecnológicas e inovadoras para os } \\
\text { problemas de Engenharia Civil. }\end{array}$ & Projetar \\
\hline A9 & $\begin{array}{l}\text { Acompanhar o ritmo de desenvolvimento técnico-científico do setor e adaptar-se } \\
\text { as constantes transformações estimulando o aprendizado autônomo. }\end{array}$ & Desenvolver-se \\
\hline ID & Habilidades transversais & Palavra-chave \\
\hline B1 & Planejar e gerir estrategicamente & Estratégia \\
\hline B2 & Atuar em equipes multidisciplinares & Equipe \\
\hline B3 & Comunicação (escrita, oral e gráfica) & Comunicar-se \\
\hline B4 & Exercer a cidadania como engenheiro \\
\hline
\end{tabular}

${ }^{1}$ Identificação definida para a competência.

Fonte: Os autores (2021).

Para verificar quais competências estavam sendo desenvolvidas no curso propôs-se ao grupo identificar os objetivos de aprendizado de cada disciplina agrupadas por temas gerais. Posteriormente, esses objetivos de aprendizado foram correlacionados com as competências que estes tinham por alvo desenvolver. Como exemplo apresentamos no Quadro 3 os objetivos de aprendizagem das disciplinas de Mecânica dos Fluidos e Hidráulica II. 
28 a 30 de SETEMBRO

Quadro 3 - Exemplificação da ligação entre as competências e os objetivos de aprendizagem.

\begin{tabular}{|c|c|c|c|}
\hline \multicolumn{4}{|c|}{ Tema geral: Águas } \\
\hline Disciplinas & Objetivos de aprendizagem & Com & ncias \\
\hline \multirow{7}{*}{$\begin{array}{l}\text { Mecânica dos } \\
\text { Fluidos }\end{array}$} & - Obter noções fundamentais dos fluidos; & A1 & A2 \\
\hline & - Desenvolver a análise dimensional de fórmulas; & A1 & A3 \\
\hline & - Compreender o procedimento de semelhança mecânica; & A1 & A3 \\
\hline & - Calcular esforços hidrostáticos sobre superfícies; & A1 & A2 \\
\hline & - Conhecer os conceitos de cinemática dos fluidos; & A1 & $\mathrm{A} 2$ \\
\hline & - Conhecer o equacionamento básico de dinâmica dos fluidos; & A1 & $\mathrm{A} 2$ \\
\hline & - Calcular escoamentos viscosos em condutos. & A1 & $\mathrm{A} 2$ \\
\hline \multirow{6}{*}{ Hidráulica II } & $\begin{array}{l}\text { - Conhecer os conceitos fundamentais de escoamentos com } \\
\text { superfície livre; }\end{array}$ & A1 & $\mathrm{A} 2$ \\
\hline & $\begin{array}{l}\text { - Aplicar o equacionamento de escoamentos uniformes, } \\
\text { compreendendo os aspectos de projeto; }\end{array}$ & A1 & A2 \\
\hline & - Analisar o escoamento com relação a sua energia específica; & \multicolumn{2}{|c|}{ A2 } \\
\hline & $\begin{array}{l}\text { - Conhecer e aplicar os cálculos básicos sobre ressaltos } \\
\text { hidráulicos; }\end{array}$ & A1 & A2 \\
\hline & $\begin{array}{l}\text { - Conhecer e aplicar os cálculos básicos sobre movimentos } \\
\text { gradualmente variados; }\end{array}$ & A1 & A2 \\
\hline & $\begin{array}{l}\text { - Compreender os conceitos básicos sobre escoamentos } \\
\text { bruscamente variados. }\end{array}$ & A1 & $\mathrm{A} 2$ \\
\hline
\end{tabular}

Fonte: Os autores (2021).

Como não existe nenhuma indicação no PPC sobre a metodologia a ser utilizada para cada disciplina, não existe a denotação que disciplinas como Mecânica dos Fluidos e Hidráulica II desenvolvam soft skills, mesmo que uma metodologia ativa tenha sido utilizada na disciplina, pois isso é algo que depende exclusivamente do professor e não está definido no PPC vigente.

\subsection{Recorrência das competências nas atividades}

O procedimento de inferência de objetivos de aprendizagem por disciplina foi realizado para todas as 57 atividades do curso (disciplinas e estágio obrigatório) com a colaboração dos professores responsáveis pelas mesmas e, então, obteve-se a recorrência com que cada competência foi relacionada a um objetivo de aprendizado (Quadro 4), levando em consideração a carga horária da disciplina de acordo com a Equação (1),

$$
C=\frac{\sum\left(\frac{C O A}{N O A} \times C H\right)}{C H_{\text {total }}}
$$

onde: $\mathrm{C}$ é a relação unitária de determinada competência para uma atividade (disciplina/estágio), COA é o número de vezes que a determinada competência é 
beneficiada pelos objetivos de aprendizado específicos da atividade, NOA é a quantidade de vezes que todas as competências são beneficiadas por cada objetivo de aprendizado específico da atividade, $\mathrm{CH}$ é carga horária da atividade em questão e $\mathrm{CH}_{\text {total }}$ é a carga horária total do curso, descontando as atividades complementares. A equação apresentada possui duas hipóteses que são: cada competência é aprendida de forma separada, requerendo uma carga horária própria para ela e; os objetivos de aprendizado são ensinados igualitariamente em carga horária para uma determinada atividade. Mesmo que os pesquisadores saibam das simplificações acarretadas pelas hipóteses, esta foi a alternativa encontrada para quantificar o desenvolvimento das competências ao longo do curso com os dados disponíveis para o estudo. Os resultados são apresentados no Quadro 4.

Quadro 4 - Recorrência das competências nos objetivos de aprendizagem para todo o curso.

\begin{tabular}{|c|c|c|c|}
\hline$\%$ & ID & Competências gerais do curso & Palavra-chave \\
\hline $40 \%$ & A1 & Aprender e aplicar conhecimentos de matemática, ciência e engenharia. & Conhecer \\
\hline $4 \%$ & $\mathrm{~A} 2$ & $\begin{array}{l}\text { Analisar e compreender fenômenos físicos e químicos inerentes a } \\
\text { Engenharia Civil. }\end{array}$ & Compreender \\
\hline $3 \%$ & A3 & Projetar e conduzir experimentos, bem como analisar e interpretar dados. & Investigar \\
\hline $21 \%$ & A4 & Formular, analisar e resolver problemas de Engenharia Civil. & Resolver \\
\hline $6 \%$ & A5 & $\begin{array}{l}\text { Conceber soluções de Engenharia Civil, analisando e compreendendo a } \\
\text { necessidade dos usuários e seu contexto. }\end{array}$ & Solucionar \\
\hline $4 \%$ & A6 & Implantar, supervisionar e controlar as soluções de Engenharia Civil. & Executar \\
\hline $4 \%$ & A7 & $\begin{array}{l}\text { Conhecer e aplicar com ética a legislação e os atos normativos } \\
\text { compreendendo o impacto das soluções nos setores global, econômico, } \\
\text { ambiental e contexto social. }\end{array}$ & Legislação \\
\hline $1 \%$ & A8 & $\begin{array}{l}\text { Criar, projetar e implementar soluções tecnológicas e inovadoras para os } \\
\text { problemas de Engenharia Civil. }\end{array}$ & Projetar \\
\hline $1 \%$ & A9 & $\begin{array}{l}\text { Acompanhar o ritmo de desenvolvimento técnico-científico do setor e } \\
\text { adaptar-se as constantes transformações estimulando o aprendizado } \\
\text { autônomo. }\end{array}$ & Desenvolver-se \\
\hline$\%$ & ID & Habilidades transversais & Palavra-chave \\
\hline $4 \%$ & B1 & Planejar e gerir estrategicamente & Estratégia \\
\hline $4 \%$ & $\mathrm{~B} 2$ & Atuar em equipes multidisciplinares & Equipe \\
\hline $3 \%$ & B3 & Comunicação (escrita, oral e gráfica) & Comunicar-se \\
\hline $3 \%$ & B4 & Exercer a cidadania como engenheiro & Cidadania \\
\hline
\end{tabular}

${ }^{1}$ Identificação definida para a competência.

Fonte: Os autores (2021).

É possível observar que grande parte das competências desenvolvidas dentro das atividades estão voltadas para o conhecimento básico, como é o caso das competências A1, A2, A3 e A4 que juntas correspondem a $68 \%$ da recorrência das competências nos objetivos de aprendizado, mesmo que apenas $30 \%$ da carga horária do curso seja destinada a disciplinas de formação básica (como apresentado no Quadro 1).

Ainda, a alta porcentagem das competências A1, A2, A3, A4 e A5 representam a quantidade de conhecimentos gerais que o engenheiro civil necessita possuir para a sua 
formação. Visto que a engenharia civil possui uma área de atuação bastante ampla, se faz necessária grande carga horária para o desenvolvimento das competências relacionadas às palavras-chave citadas no Quadro 4: conhecer, compreender, investigar, resolver e solucionar. Em contrapartida, ainda existe a necessidade do desenvolvimento de soft skills, que atualmente estão sendo pouco praticadas no curso, para que o egresso possa contribuir efetivamente para a sociedade compreendendo as suas necessidades.

A relação de apenas $2 \%$ constituída pelas atividades A8 e A9 demonstram uma deficiência com relação à inovação e ao desenvolvimento científico já que são os itens relacionados a soluções tecnológicas e inovadoras e acompanhar o ritmo de desenvolvimento técnico-científico. Normalmente, estas competências são desenvolvidas em atividades complementares, como a participação do discente em atividades de pesquisa e extensão, e não nas disciplinas propriamente ditas. Entretanto, em vista do rápido crescimento técnico e científico, temas relacionados à inovação devem possuir algum tipo de incentivo nas disciplinas oferecidas, pois são temas pertinentes a qualquer engenheiro da atualidade.

\subsection{Relação das recorrências das competências com os objetivos do curso}

É possível correlacionar a recorrência do desenvolvimento das competências com os objetivos do curso (Quadro 5). Observa-se que grande parte das atividades estão voltadas para o objetivo I do curso, enquanto os outros quatro objetivos são desenvolvidos em apenas $14 \%$ da carga horária dedicada às atividades do curso. Isso evidencia que, mesmo que o PPC estivesse em consonância com as DCNs de 2002, o curso efetivamente possui uma grande discrepância com relação aos esforços efetuados para atingir os objetivos estabelecidos.

Quadro 5 - Resumo.

\begin{tabular}{|c|c|c|c|}
\hline $\begin{array}{l}\text { Objetivo } \\
\text { do curso }\end{array}$ & $\begin{array}{l}\text { Competências } \\
\text { relacionadas }^{2}\end{array}$ & Recorrência $^{2}$ & Comentário \\
\hline I & $\mathrm{A} 1-\mathrm{A} 9$ & $86 \%$ & $\begin{array}{l}\text { Este é um objetivo que potencialmente está sendo } \\
\text { desenvolvido adequadamente, pois ele se relaciona com } \\
\text { as competências estabelecidas pelo NDE. }\end{array}$ \\
\hline II & B1, B2 e B4 & $11 \%$ & \multirow{2}{*}{$\begin{array}{l}\text { Pode ser mais amplamente contemplado por } \\
\text { metodologias ativas de ensino e aprendizado. O trabalho } \\
\text { em equipe realizado pelos alunos deve considerar um } \\
\text { método adequado para maximizar o aprendizado } \\
\text { oferecido per esta experiência. }\end{array}$} \\
\hline III & B2 e B4 & $7 \%$ & \\
\hline IV & B3 & $3 \%$ & $\begin{array}{l}\text { É importante que os professores incentivem o uso de } \\
\text { ferramentas computacionais em suas disciplinas. } \\
\text { Acredita-se que as disciplinas desenvolvem } \\
\text { indiretamente estas habilidades. Cabe ao PPC } \\
\text { evidenciar este tipo de desenvolvimento para cada } \\
\text { atividade. }\end{array}$ \\
\hline V & A7 e B4 & $7 \%$ & $\begin{array}{l}\text { Existem maneiras de se dar enfoque em disciplinas mais } \\
\text { específicas nestes temas. Apresentar a } \\
\text { responsabilidade que o profissional possui, não perante } \\
\text { apenas às suas atividades, mas perante toda a } \\
\text { sociedade é necessária para a formação de um } \\
\text { profissional ativo. }\end{array}$ \\
\hline
\end{tabular}

${ }^{1}$ Proveniente do PPC de 2008. ${ }^{2}$ Definidos no Quadro 4.

Fonte: Os autores (2021).

Nota-se que os objetivos do curso relacionados às soft skills acabam por possuir competências que se repetem em outros objetivos, pois muitas vezes o desenvolvimento em uma acarreta o desenvolvimento de outra de forma sinérgica. Assim, um esforço para 
o desenvolvimento de habilidades transversais pode contemplar mais do que um objetivo do curso simultaneamente.

Ainda, se levarmos em consideração as propostas do PPC para qualidade de formação do curso, temos um resultado semelhante, em que diversas propostas estão relacionadas ao desenvolvimento de habilidades transversais como "dar ao aluno mais responsabilidade e participação no processo de aprendizagem, e; melhorar as habilidades nas relações interpessoais, de comunicação e expressão".

\section{CONSIDERAÇÕES FINAIS}

O presente estudo avaliou os objetivos de aprendizado das disciplinas obrigatórias, incluindo o estágio curricular e o Trabalho de Conclusão de Curso (TCC), e correlacionouos com as competências estabelecidas com base no PPC de 2008 do curso de Engenharia Civil da UDESC, nas DCNs de 2019, em PPCs de outras IES e em documentos de órgãos de acreditação de cursos, como a ABET.

Os resultados evidenciaram que existe pouca ocorrência do desenvolvimento de habilidades transversais, equivalente a $14 \%$ da carga horária total avaliada. É importante compreender que a verificação das competências foi realizada unicamente perante as disciplinas e estágio obrigatório, mesmo que o curso apresente uma carga horária de 9,17\% em atividades complementares e ainda existam disciplinas não obrigatórias (optativas) que não foram contabilizadas. Para o TCC a hora efetiva em atividades é maior do que o considerado em créditos na disciplina, o que pode acarretar erros na metodologia utilizada para verificar a proporção de competências desenvolvidas nesta atividade.

A universidade é um ambiente que oferece inúmeras atividades, além das obrigatórias, que proporcionam a promoção das soft skills, o aperfeiçoamento de técnicas e o desenvolvimento científico. De qualquer forma, atualmente, não existe nenhuma regulamentação que auxilie a compreender quais seriam as competências desenvolvidas nos casos de atividades complementares. Para que se garanta que os objetivos do curso estejam sendo alcançados pela comunidade acadêmica, torna-se necessária uma avaliação das competências desenvolvidas pelas atividades complementares através de uma quantificação individual por aluno.

Uma definição, ou ao menos sugestão, do tipo de metodologia de ensinoaprendizagem a ser utilizada por disciplina pode contribuir para que mais habilidades transversais sejam alcançadas no desenvolvimento das disciplinas, pois pelas definições atuais do PPC, torna-se difícil atestar que estas competências estão sendo aperfeiçoadas.

Entende-se que as observações realizadas pela presente pesquisa podem ser confirmadas em outros cursos de diferentes instituições de ensino. O excessivo enfoque nas habilidades específicas de solucionar problemas de engenharia foi um dos fatores que levou as novas DCNs a explicitarem o desenvolvimento das habilidades transversais e consequentemente a relação da universidade com o meio externo a ela.

\section{REFERÊNCIAS}

CONSELHO NACIONAL DE EDUCAÇÃO. Resolução CNE/CES n 2: Institui as

Diretrizes Curriculares Nacionais do Curso de Graduação em Engenharia. Brasília, 2019.

DE LOS RÍOS-CARMENADO, I.; LÓPEZ, F. R.; GARCÍA, C. P. Promoting Professional Project Management Skills in Engineering Higher Education: Project-based Learning (PBL) Strategy. International Journal of Engineering Education, 2015.

DEPARTAMENTO DE ENGENHARIA CIVIL UDESC/CCT. Projeto Pedagógico do 
Curso. Joinville, 2008.

KOLMOS, A. et al. Facilitation on PBL environment. UNESCO Chair in Problem Based Learning, 2008.

SCOTT, N.; HADGRAFT, R.; ILIC, V. Engineering Education - Is Problem-Based or Project-Based Learning the answer? Australasian Journal of Engineering Education, v.4, P 2-17, 2003.

\title{
ASSESSMENT OF THE COMPETENCES AND LEARNING OBJECTIVES OF THE CCT/UDESC CIVIL ENGINEERING COURSE
}

\begin{abstract}
Nowadays, it is difficult to recruit engineers who, in addition to the technical domain, have skills related to leadership, group work, planning, strategic management and that learn autonomously. The present work aims to describe the current situation of the civil engineering course of the Universidade do Estado de Santa Catarina on the Centro de Ciências Tecnológicas through the establishment of learning objectives and its relationship with the development of the needed competencies. The results show that most of the competencies developed in the activities are focused on the most basic knowledge that together correspond to $68 \%$ of the recurrence of competencies in the learning objectives, even if only $30 \%$ of the course workload is allocated to basic disciplines development. There is little occurrence of soft skills development, which is equivalent to $14 \%$ of the total workload evaluated. It is understood that the observations made by the present research can be confirmed in other degree courses of different educational institutions.
\end{abstract}

Keywords: Education in Engineering. National Curriculum Guidelines. Competences. 